\title{
Early and late insulin response as predictors of NIDDM in Pima Indians with impaired glucose tolerance
}

\author{
D. K. Nagi, W. C. Knowler, M. A. Charles, Q.Z.Liu, R.L.Hanson, D. R. McCance, D. J.Pettitt, P.H. Bennett \\ Diabetes and Arthritis Epidemiology Section, Phoenix Epidemiology and Clinical Research Branch, National Institute of \\ Diabetes and Digestive and Kidney Diseases, Phoenix, Arizona, USA
}

Summary Risk factors predicting deterioration to diabetes mellitus were examined in 181 subjects with impaired glucose tolerance. Fifty-seven subjects had impaired glucose tolerance on one occasion followed by normal glucose tolerance at a repeat oral glucose tolerance test, and 124 subjects had impaired glucose tolerance on two successive oral glucose tolerance tests. Subjects were followed for a median period of 5.0 years (range 1.0-17.2). The age- and sex-adjusted cumulative incidence of diabetes at 10 years of follow-up was higher in subjects who had impaired glucose tolerance on both tests $(70 \%)$ than in those whose glucose tolerance was normal at the repeat test $(53 \%)$, [rate ratio $(\mathrm{RR})=1.6,95 \%$ confidence intervals $(\mathrm{CI})=1.0-2.5]$. Proportional hazards analyses were used to identify baseline risk factors (measured at the repeat oral glucose tolerance test) for subsequent diabetes, and incidence rate ratios were calculated for the 90th percentile compared with the 10 th percentile of each continuous variable for the whole group. In all subjects, in separate models, higher body mass index $[\mathrm{RR}=2.0,95 \%$
$\mathrm{CI}=2.2-9.9]$, high fasting serum insulin concentrations $[\mathrm{RR}=2.4,95 \% \mathrm{CI}=1.4-4.2]$, and low early insulin response $[\mathrm{RR}=0.5,95 \% \mathrm{CI}=0.3-0.8] 30 \mathrm{~min}$ after a glucose load were significant predictors for deterioration to diabetes. In a multivariate analysis which controlled for age and sex, 120-min post-load glucose, fasting insulin and late insulin response predicted diabetes. In subgroup analyses the predictors of diabetes were generally similar in subjects who had impaired glucose tolerance at only one test and those who had impaired glucose tolerance on both tests. These findings suggest that in those subjects with impaired glucose tolerance whose glucose tolerance has returned to normal, the risk of subsequent diabetes is high. Insulin resistance, impaired early insulin response, or both, are predictive of subsequent development of diabetes in Pima Indians with impaired glucose tolerance. [Diabetologia (1995) 38: 187-192]

Key words Impaired glucose tolerance, insulin response, cholesterol.
Previous studies in Pima Indians and other populations have shown that subjects with impaired glucose tolerance (IGT), defined on the basis of a single oral glucose tolerance test (OGTT), are at high risk for subsequent diabetes mellitus $[1,2]$, and that this risk

Received: 20 June 1994

Corresponding author: Dr. D. K. Nagi, Department of Endocrinology, General Infirmary at Leeds, 1 Great George Street, Leeds, LS1 3EX, UK

Abbreviations: IGT, Impaired glucose tolerance; OGTT, oral glucose tolerance test; NGT, normal glucose tolerance; CV, coefficient of variation. is considerably lower in subjects in whom IGT is transient [3]. Subjects with IGT are also at increased risk of macrovascular disease $[1,4]$. Whether excess risk of macrovascular disease is also shared by subjects in whom IGT is transient is unknown. Obesity and higher fasting and lower 120-min serum insulin levels following an oral glucose load are risk factors for subsequent diabetes in Pima Indians with IGT [2]. A comparison of the early and late insulin responses as predictors of diabetes in Pima Indians has not been published previously.

In the present study we have identified two groups of subjects with IGT: (i) those who had IGT on two 
consecutive examinations, and (ii) those who had IGT at one examination but normal glucose tolerance (NGT) at the second.

The aims of this investigation were:

(i) to identify risk factors for progression to diabetes in these two IGT groups, with particular emphasis on the early and the late insulin response during the OGTT

(ii) to see if the risk factors for diabetes differ between the two groups.

\section{Subjects, materials and methods}

Subjects have been studied in an ongoing epidemiologic study of non-insulin-dependent diabetes (NIDDM) among the Pima Indian residents in the Gila River Indian Community in Central Arizona. Subjects are examined biennially from age 5 years, including a medical history and physical examination [5]. The subjects also undergo a modified OGTT, with venous plasma collected at fasting and at 120 min after a 75 -g carbohydrate load. The diagnosis of diabetes $(120$-min plasma glucose $\geq 11.1 \mathrm{mmol} / \mathrm{l})$ and of IGT (120-min plasma glucose $\geq 7.8$ but $<11.1 \mathrm{mmol} / \mathrm{l}$ ) was made by World Health Organisation (WHO) criteria [6]. Subjects in whom the 120 -min plasma glucose is more than $8.9 \mathrm{mmol} / \mathrm{l}$ and less than $11.1 \mathrm{mmol} / \mathrm{l}$ are requested to return for a repeat OGTT. At that time blood is collected after an overnight fast and at 30,60 and 120 min after the glucose load.

This study includes subjects who had a repeat OGTT between February 1973 and June 1986. We have presented data during this period because insulin assays were performed using a single method. As one of the main aims of the study was to compare early and late insulin response, data are presented only from those subjects in whom results of 30 - and $120-\mathrm{min}$ post-load insulin concentrations were available. All subjects, with no previous known glucose tolerance abnormality were selected at the time when they were first noted to have IGT, and then subsequently underwent a repeat examination.

A repeat OGTT was performed on 213 subjects with IGT. Follow-up data were available for 181 subjects. IGT on two consecutive tests was identified in 124 subjects and IGT on the first but NGT on the second test was identified in 57 subjects. The median duration between the two OGTTs was 49 days (range 9-469) for those who had IGT on both tests and 56 days (range 14-371) in those who had IGT on the first but not on the second test. These subjects were followed-up for the subsequent development of diabetes. The period of risk started from the day of the repeat OGTT. The end point of the study was either the development of diabetes or the last biennial examination for those who did not develop diabetes. Plasma glucose was measured by the modified method of Hoffman [7]. Serum insulin concentrations measured by radioimmunoassay using a double antibody method [8] with an interassay $\mathrm{CV}$ of $6-8 \%$, were reported in $\mu \mathrm{U} / \mathrm{ml}$ and converted to $\mathrm{pmol} / \mathrm{l}(1 \mu \mathrm{U} / \mathrm{ml}=6.0 \mathrm{pmol} / \mathrm{l})$.

\section{Statistical analysis}

Glucose and insulin concentrations were approximately lognormally distributed so their logarithms were used in statistical analysis. Insulin responses were defined as the post-load insulin concentrations relative to the fasting insulin concentrations. Thus, the "early insulin response" during the OGTT was expressed as the ratio of the 30 -min to fasting insulin concentrations and the "late insulin response" as the ratio of the 120 min to fasting insulin concentrations. The natural logarithms of these ratios were used to normalise their distributions. The effects of baseline risk factors, i.e. risk factors measured at the time of the repeat OGTT, on the subsequent development of diabetes were analysed by Cox's proportional hazards analysis [9]. Age, body mass index, fasting and post-load plasma glucose, fasting and post-load insulin concentrations, and early and late insulin responses, were examined for their ability to predict subsequent diabetes. Variables were tested individually to identify those predictive of diabetes, and also in a stepwise model with age and sex forced into the model.

The cumulative incidence of diabetes, adjusted for age and sex, was calculated in each group from the survival function estimate derived from the proportional hazards model. Cumulative incidence of diabetes was also calculated according to early and late insulin response using separate proportional hazards models. Both early and late insulin responses were first adjusted for 120 -min plasma glucose by linear regression. Subjects whose insulin responses were above that predicted were arbitrarily defined as those with high insulin responses while those with insulin responses below that predicted as those with low insulin response. In models including subjects who had IGT on only one test and those who had IGT on both tests, interaction terms were used to assess whether risk factors for predicting diabetes differed between the two groups. Rate ratios (RR) are shown comparing 90th with the 10 th percentile of continuous variables.

\section{Results}

Clinical details of subjects at the first and at the repeat OGTT for both IGT groups are shown in Table 1 . Subjects who had IGTon one test only were significantly younger and had lower 120 -min plasma glucose than subjects with IGT on both tests. Fasting and 120-min insulin concentrations were similar in the two groups. In subjects who had IGT on one test, the body mass index did not differ substantially between the two examinations, but as expected, inherent in the selection criteria, 120-min glucose concentrations were lower at the repeat examination, as were the fasting glucose concentrations. Subjects in this group also had significantly lower 120 -min, but not fasting, serum insulin concentrations at the repeat examination. Serum cholesterol concentrations were also significantly lower at the repeat examination in this group.

In subjects with IGT on both tests, fasting and 120 min glucose concentrations were both significantly lower at the repeat OGTT. These changes in plasma glucose were accompanied by significant lowering of fasting insulin but not of 120 -min insulin concentrations. These was little change in the serum cholesterol concentration from first to the second test in these subjects.

In all subjects, fasting insulin concentrations measured at the repeat OGTT were significantly and positively correlated with $30-\mathrm{min}\left(r_{\mathrm{s}}=0.53, p=0.0001\right)$, $60-\min \left(r_{\mathrm{s}}=0.57, p=0.0001\right)$ and $120-\min \left(r_{\mathrm{s}}=0.47\right.$, 
Table 1. Clinical characteristics of subjects with impaired glucose tolerance (IGT)

\begin{tabular}{|c|c|c|c|c|}
\hline \multirow[t]{2}{*}{ Variables } & \multicolumn{2}{|c|}{ IGT on one test $(n=57)$} & \multicolumn{2}{|c|}{ IGT on both tests $(n=124)$} \\
\hline & First OGTT & Repeat OGTT & First OGTT & Repeat OGTT \\
\hline Age (years) & \multicolumn{2}{|l|}{$30.3(13.2,51.4)$} & \multicolumn{2}{|l|}{$34.8(16.7,60.3)^{\mathrm{a}}$} \\
\hline Systolic blood pressure (mm Hg) & $119 \pm 18$ & - & $123 \pm 18$ & - \\
\hline Diastolic blood pressure (mm Hg) & $74 \pm 13$ & - & $74 \pm 13$ & - \\
\hline Total cholesterol (mmol/1) & $4.5 \pm 0.9$ & $4.3 \pm 0.7^{\mathrm{c}}$ & $4.3 \pm 0.8$ & $4.2 \pm 0.8$ \\
\hline $60-\mathrm{min}$ glucose $(\mathrm{mmol} / \mathrm{l})$ & - & $9.1 \pm 1.9$ & - & $10.9 \pm 1.6$ \\
\hline $120-\mathrm{min}$ glucose $(\mathrm{mmol} / 1)$ & $9.5 \pm 0.6$ & $6.6 \pm 0.9^{b}$ & $9.8 \pm 0.6^{\mathrm{a}}$ & $9.4 \pm 0.9^{c}$ \\
\hline Fasting insulin (pmol/1) & $249(102,471)$ & $222(108,463)$ & $255(102,458)$ & $210(110,408)^{c}$ \\
\hline $30-\mathrm{min}$ insulin $(\mathrm{pmol} / 1)$ & - & $918(406,2360)$ & - & $879(465,2058)$ \\
\hline $60-\mathrm{min}$ insulin $(\mathrm{pmol} / 1)$ & - & $1215(601,2809)$ & - & $1140(588-2685)$ \\
\hline $120-\min$ insulin $(\mathrm{pmol} / \mathrm{l})$ & $1620(672,4299)$ & $960(258,2100)^{c}$ & $1434(684-3043)$ & $1374(576,2856)$ \\
\hline \multicolumn{5}{|c|}{$\begin{array}{l}\text { Variables are given as mean } \pm \text { SD or median (10th, } 90 \text { th per- } \\
\text { centile) } \\
\text { Significance of difference between variables at two oral glu- } \\
\text { cose tolerance tests (OGTT) was analysed by Students' paired }\end{array}$} \\
\hline
\end{tabular}

Table 2. Predictors of subsequent development of diabetes in subjects with IGT

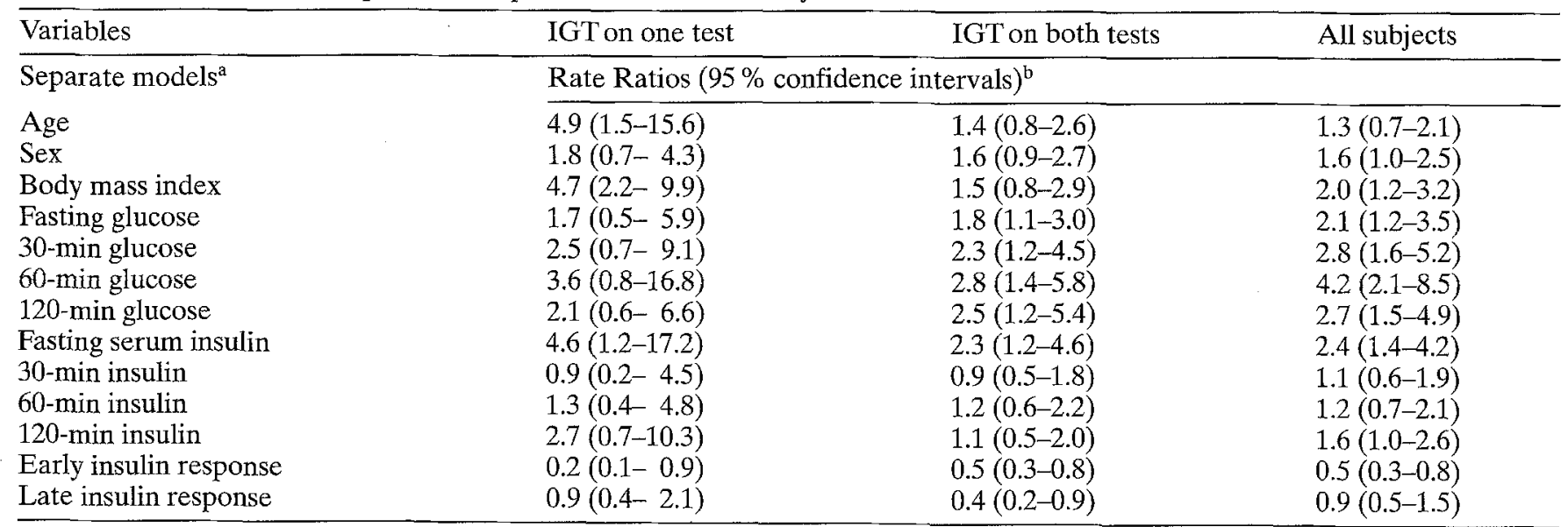

${ }^{a}$ Age and sex included in each separate model. Because of missing data, the numbers included in each model varied; ${ }^{b} \mathrm{Rate}$ ratios $(95 \% \mathrm{CI})$ are shown comparing the 90 th percentile with the 10 th percentile

$p=0.0001)$ insulin concentrations, but were negatively correlated with both early $\left(r_{\mathrm{s}}=-0.38, p=0.0001\right)$ and late $\left(r_{\mathrm{s}}=-0.25, p=0.001\right)$ insulin response.

Of 124 subjects who had IGT on both tests, 75 $(60 \%)$ developed diabetes after a median follow up of 5.0 years (range $1.0-17.2), 34(27 \%)$ continued to have IGT, and $15(12 \%)$ reverted to NGT. In subjects with IGT on one test, $25(44 \%)$ developed diabetes after a median follow up 4.4 years (range 1.415.3), $20(35 \%)$ continued to have NGT and 12 (21\%) developed IGT again.

The cumulative incidence of diabetes during 10 years of follow-up, adjusted for age and sex, is shown in Figure 1 for both groups. Subjects with IGT on both tests had a higher cumulative incidence of diabetes than subjects with $\mathrm{IG}^{\mathrm{T}} \mathrm{T}$ on one test alone $[\mathrm{RR}=1.6,95 \% \mathrm{CI}=1.0-2.5]$.

In a proportional hazards model, which included all subjects, age was not a significant predictor of diabetes, while male sex was a positive predicter. Adjusted for age and sex, body mass index was a significant predictor of diabetes. In all subjects, high fasting and 120-min insulin concentration, low early insulin response, fasting and post-load glucose concentrations, were each predictive of diabetes in separate models, while $30-\mathrm{min}$ and 60 -min insulin concentrations and late insulin response were not (Table 2).

With age and sex forced into the stepwise models, 120-min glucose, fasting insulin concentration and 
Table 3. Results of multivariate analyses for predicting diabetes in subjects with IGT

\begin{tabular}{|c|c|}
\hline Variables & $\begin{array}{l}\text { Rate ratios } \\
(95 \% \text { confidence intervals })^{b}\end{array}$ \\
\hline \multicolumn{2}{|c|}{$\begin{array}{l}\text { All subjects }\left(n=169^{c} \text {, new cases of diabetes }=94\right) \\
\text { Model }^{\mathrm{a}}\end{array}$} \\
\hline $120-$ min glucose & $2.1(1.4-3.1)$ \\
\hline Fasting insulin & $2.1(1.1-3.7)$ \\
\hline Late insulin response & $0.5(0.3-0.9)$ \\
\hline \multicolumn{2}{|c|}{$\begin{array}{l}\text { IGT on one test }\left(n=56^{\mathrm{c}} \text {, new cases of diabetes }=25\right) \\
\text { Model }^{\mathrm{a}}\end{array}$} \\
\hline Fasting insulin & $4.0(1.2-13.6)$ \\
\hline \multicolumn{2}{|c|}{$\begin{array}{l}\text { IGT on both tests }\left(n=113^{\mathrm{c}}, \text { new cases of diabetes }=69\right) \\
\text { Model }^{\mathrm{a}}\end{array}$} \\
\hline Early insulin response & $0.5(0.3-0.8)$ \\
\hline
\end{tabular}

${ }^{a}$ Age, sex, body mass index, fasting and 120-min insulin concentrations, early and late insulin response, fasting and 120min plasma glucose concentrations available in the model. Age and sex were forced into the model. ${ }^{b}$ Rate ratios $(95 \%$ confidence intervals) shown comparing 90th percentile with the 10th percentile. ${ }^{\mathrm{c}}$ Number of subjects with data available on all variables

late insulin response were selected as the best predictors of diabetes, with no other variable contributing significantly (Table 3 ). In subjects with IGT on one test, in a similar analysis, higher fasting insulin concentration was selected as the only significant predictor of diabetes. In subjects with IGT on both tests, lower early insulin response was selected as the only significant predictor of diabetes (Table 3).

The cumulative incidence of diabetes according to early (Fig. 2a) and late insulin responses (Fig. 2 b) (adjusted for 120-min plasma glucose in a linear regression model) is shown. In all subjects, those with a lower early insulin response had a higher cumulative incidence of diabetes, and the effect of early insulin response on future development diabetes was more pronounced at a lower 120 -min glucose concentrations (significant interaction of early insulin response at 120 -min glucose concentration, $p<0.05$ ). Those with a low late insulin response, when stratified by 120 -min glucose, had a slightly, but not significantly, higher incidence of diabetes.

Subgroup analyses showed that in subjects who had IGT on both tests, low early and late insulin responses and high fasting and post-load glucose concentrations, in separate models, were predictive of diabetes, while post-load insulin concentrations were not. Age was a significant predictor of diabetes in subjects who had IGT on one test but not in those with IGT on both tests, although the latter group was on average somewhat older at the first examination. Body mass index was a significant predictor of diabetes in those with IGT on one test but not in those who had IGT on both tests (Table 2).

The observed differences in age and late insulin response for predicting diabetes in the two groups were

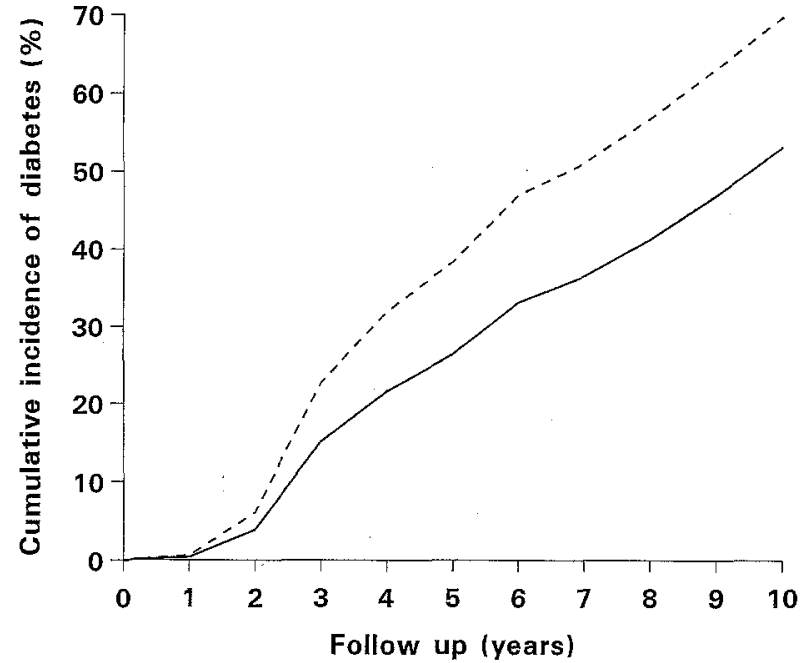

Fig. 1. Cumulative incidence of diabetes over 10 years, adjusted for age and sex in subjects with IGT on one test (-) and IGT on both tests (-..--)

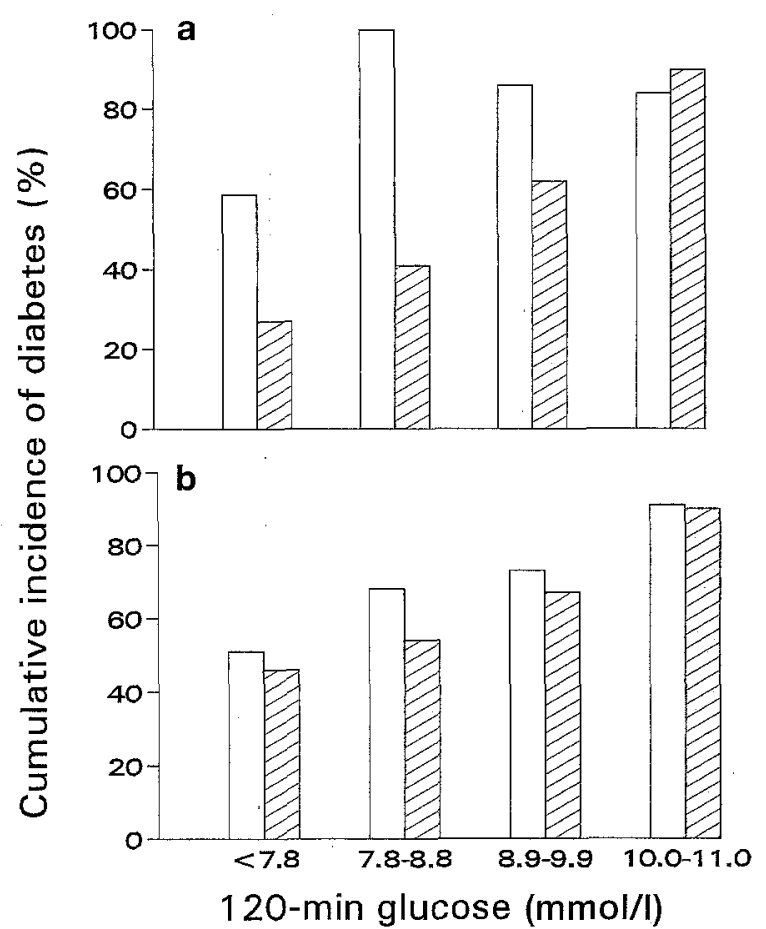

Fig. 2 (a,b). 10-year cumulative incidence of diabetes according to early (a) and late (b) insulin response. Early and late insulin response were adjusted for 120 -min plasma glucose in a linear regression model in all subjects. $\square$ low; $\varangle$ high

analysed further. The interaction terms of age and late insulin response, with IGT on one test or on both tests as a group variable, were included in analysis of all subjects in a single model. Both interaction terms were statistically significant $(p<0.05)$ indicating that these two risk factors had different effects in these two groups. Older age was positively associated with diabetes in those who had IGT on one test but not in those who had IGT on both tests. Converse- 
ly a late insulin response was negatively associated with diabetes in subjects who had IGT on both tests but not in those who had NGT on the second test.

\section{Discussion}

Suggestions that subjects with IGT may be a target group for intervention aimed at preventing NIDDM $[10,11]$ seem particularly relevant to Pima Indians, who have the world's highest recorded incidence of NIDDM [12]. For such an intervention to be successful, criteria for the selection of subjects with IGT who are likely to develop diabetes are important.

In this study the cumulative incidence of diabetes was higher in subjects with IGT on two tests than in subjects who had IGT on only one test but NGT on the repeat test, indicating that subjects selected to have IGT on the basis of two OGTTs have a higher risk of developing diabetes than subjects with IGT diagnosed on the basis of one OGTT, as reported previously [3].

It is also evident from the present data that some risk factors for subsequent diabetes are somewhat different in subjects with IGT diagnosed on the basis of one or two tests. Although older age has been shown to be a risk factor for diabetes in Caucasian subjects [13], previous studies in the Pima Indians have shown that age-specific incidence rates are highest between 35 and 54 years, either among all subjects [5] or among those with IGT [2]. The lack of association of age with subsequent diabetes in subjects with IGT on two tests, compared with a strong predictive ability of age in those with IGT on one test and not on the second, could be due to the younger age of subjects in the latter group. The subjects with IGT on one test were in the younger age range, where the incidence of diabetes is positively related to age, and those with IGT on two tests were in the age range where the age-specific incidence curve is flatter. This observation also suggests that IGT diagnosed on the basis of a single test represents an earlier stage in development of NIDDM.

Previous studies have shown obesity to be a risk factor for diabetes in normal subjects and in subjects with IGT $[2,14,15,17]$ and the results of our study are similar. However, body mass index controlled for age and sex, was not predictive of subsequent diabetes in subjects with IGT on both tests, suggesting that obesity may no longer be an important risk factor for subsequent diabetes after IGT has been established.

Subjects with NIDDM are insulin resistant and have impaired insulin secretion [18]. Pima Indians with IGT have impaired insulin action [19]. A recent study has reported that subjects with IGT have diminished early insulin response due to beta-cell dysfunction and that this diminished early insulin response and the consequent relative hyperglycaemia may be responsible for hyperinsulinaemia seen 120 min after an oral glucose load [20]. Nevertheless the higher fasting insulin concentrations which characterise subjects with IGT appear to be the result of impaired insulin action at the target cell level $[19,21,22]$.

In all subjects, early but not late insulin response was a significant predictor of diabetes, findings which are similar to those reported in Japanese subjects with IGT [16]. However, subgroup analyses showed that in subjects who had IGT on two tests, diminished early and late insulin responses were both predictive of diabetes, but this was not the case in subjects who had IGT on one test, in whom only low early insulin response was predictive. These findings suggest that subjects with IGT who revert to normal at the repeat test have an earlier abnormality of glucose homeostasis prior to developing a more severe or persistent form of glucose intolerance, represented in this study by a group of subjects who had IGT on the first and the repeat test.

Whether or not a diminished early insulin response in subjects with normal glucose tolerance leads to the development of IGT, however, cannot be answered by this study, because the early insulin response was measured only in subjects who had IGT on the first examination. However, it is possible that the diminished early insulin response may eventually lead to more severe and persistent glucose intolerance. An alternative intepretation of these results is that in subjects with IGT, a group with high risk for future diabetes, having high early and late insulin responses seems to confer relative protection from development of diabetes for a number of years.

In subjects with IGT on two tests, high fasting insulin concentrations and low early insulin response were the most important predictors of diabetes. This finding suggests that increased insulin resistance (high fasting insulin concentration) and overall reduction in beta-cell response to carbohydrate load (lower post-load insulin relative to fasting insulin despite relative hyperglycaemia) are strong predictors of diabetes. This reduced beta-cell response may represent a smaller beta-cell mass in subjects genetically predisposed to NIDDM, a gradual beta-cell exhaustion, or possibly a "glucotoxic effect" on betacell function [23, 24].

Significant lowering of post-load insulin concentration and late insulin response with only a small change in fasting insulin concentrations would suggest that the reversion from IGT to normal glucose tolerance is due to insulin action which in turn is accompanied by changes in insulin secretory pattern. Due to the close interactive glucose-insulin feedback loop it is difficult to infer whether changes in insulin secretion were primary or secondary to changes in glucose tolerance. Previous studies have suggested that the transition from IGT to NGT may be due to 
intraindividual variability in glucose tolerance [25, $26]$, which is due to variation in glucose absorption from the gut, and uptake and utilization of glucose at the splanchnic and peripheral level [27].

In conclusion, subjects with IGT who revert to normal on repeat glucose testing are at lesser risk of diabetes than subjects with IGT on both tests. The severity of glucose tolerance, higher fasting insulin (reflecting insulin resistance) as well as a lower insulin response are strong predictors of diabetes. The definition of IGT based on the results of two OGTTs thus represents a logical way to select subjects for intervention studies because of the higher risk of diabetes associated with this group. Efforts to prevent deterioration to NIDDM in the Pima Indians and other populations with glucose intolerance should focus on ways to reduce adiposity and insulin resistance, and to improve the early insulin response.

Acknowledgements. We thank the Gila River Indian Community for its help and co-operation and the laboratory and data processing staff for their technical assistance. DKN was supported by a BDA-ICI Transatlantic fellowship.

\section{References}

1. O'Sullivan JB, Mahan CB (1968) Prospective study of 352 young patients with chemical diabetes. $N$ Engl J Med 278: 1038-1041

2. Saad MF, Knowler WC, Pettitt DJ, Nelson RG, Mott DG, Bennett PH (1988) The natural history of glucose intolerance in the Pima Indians. N Engl J Med 319: 1500-1506

3. Saad MF, Knowler WC, Pettitt DJ, Nelson RG, Bennett PH (1988) Transient impaired glucose tolerance in Pima Indians: is it important? BMJ 297: 1438-1441

4. Fuller JH, Shipley MJ, Rose G, Jarrett RJ, Keen H (1980) Coronary heart disease and impaired glucose tolerance. Lancet i: $1373-1376$

5. Knowler WC, Bennett PH, Hamman RF, Miller M (1978) Diabetes incidence and prevalence in Pima Indians: a 19 fold greater incidence than in Rochester, Minnesota. Am J Epidemiol 108: 497-505

6. World Health Organisation. (1985) Diabetes mellitus Tech Rep Ser 1985: No 727, Geneva p 9-17

7. Hoffman US (1937) A rapid photoelectric method for the determination of glucose in blood and urine. J Biol Chem 120: 51

8. Herbert V, Lau K, Gottlieb CW, Bleicher SJ (1965) Coated charcoal immunoassay of insulin. J Clin Endocrinol Metab 25: $1375-1384$

9. Cox DR (1972) Regression models and life tables. Journal of the Royal Statistical Society. Series B 34: 187-220

10. Bennett PH (1985) Impaired glucose tolerance - a target for intervention? Arteriosclerosis 5: 315-317
11. Tuomilehto J, Knowler WC, Zimmet P (1992) Primary prevention of non-insulin-dependent diabetes mellitus. Diabetes Metab Rev 8: 339-353

12. Knowler WC, Pettitt DJ, Savage PJ, Bennett PH (1981) Diabetes incidence in Pima Indians: contribution of obesity and parental diabetes. Am J Epidemiol 113: 144-156

13. Palumbo PJ, Elveback LR, Chu CP, Connolly DC, Kurland LT (1976) Diabetes mellitus: incidence, prevalence, survivorship, and causes of death in Rochester, Minnesota. 1945-1970. Diabetes 25: 566-573

14. Keen H, Jarrett RJ, McCartney P (1982) The ten-year follow-up of the Bedford survey (1962-1972): glucose tolerance and diabetes. Diabetologia 22: 73-78

15. Sasaki A, Suzuki T, Horiuchi N (1982) Development of diabetes in Japanese subjects with impaired glucose tolerance: a seven year follow-up study. Diabetologia 22: 154-157

16. Kadowaki T, Miyaki Y, Hagura R et al. (1984) Risk factors for worsening to diabetes in subjects with impaired glucose tolerance. Diabetologia 26: 44-49

17. King H, Zimmet P, Raper LR, Balkau B (1984) The natural history of impaired glucose tolerance in the Micronesian population of Nauru: a 6-year follow-up study. Diabetologia 26: 39-43

18. DeFronzo RA (1992) Pathogenesis of type 2 (non-insulindependent) diabetes mellitus: a balanced overview. Diabetologia 35: 389-397

19. Lillioja S, Mott DM, Howard BV et al. (1988) Impaired glucose tolerance as a disorder of insulin action: longitudinal and cross sectional studies in Pima Indians. N Engl J Med 318: $1217-1225$

20. Mitrakou A, Kelley D, Mokan M et al. (1992) Role of reduced suppression of glucose production and diminished early insulin release in impaired glucose tolerance. $N$ Engl J Med 326: 22-29

21. Saad MF, Knowler WC, Pettitt DJ, Nelson RG, Mott DM, Bennett PH (1989) Sequential changes in serum insulin concentration during the development of non-insulin-dependent diabetes. Lancet I: 1356-1359

22. Reaven GM, Hollenbeck CB, Chen Y-DI (1989) Relationship between glucose tolerance, insulin secretion, and insulin action in non-obese individuals with varying degree of glucose tolerance. Diabetologia 32: 52-55

23. Rossetti L, Giaccari A, DeFronzo RA (1990) Glucose toxicity. Diabetes Care 13: 610-630

24. Unger RH, Grundy S (1985) Hyperglycaemia as an inducer as well as a consequence of impaired islet cell function and insulin resistance: implications for management of diabetes. Diabetologia 28: 119-121

25. Ganda OP, Day JL, Soeldner JS, Connon JJ, Gleason RE (1978) Reproducibility and comparative analysis of repeated intravenous and oral glucose tolerance tests. Diabetes 27: $715-725$

26. Olefesky JM, Reaven GM (1971) Insulin and glucose response to identical oral glucose tolerance tests performed forty-eight hours apart. Diabetes 23: 449-453

27. Harding PE, Oakley NW, Wynn V (1973) Reproducibility of oral glucose tolerance data in normal and mildly diabetic subjects. Clin Endocrinol (Oxf) 2: 387-395 